\title{
6 x 20Gbps Long Reach WDM-PI based High Altitude Platform Inter-Satellite Communication System
}

\author{
Sushank Chaudhary \\ Optical Technology Group \\ InterNetworks Research Lab, \\ UUM Malaysia.
}

\author{
Abhishek Sharma \\ Department of Electronics and \\ Communication, \\ Beant College of Engineering and \\ Technology, Gurdaspur
}

\author{
Neetu \\ Department of Electronics and \\ Communication, \\ Beant College of Engineering and \\ Technology, Gurdaspur
}

\begin{abstract}
InterSatellite communication is one of the remarkable technologies in the era of high altitude platform (HAP) communication system. This work is focused on the transmission of six channels, each carrying 20Gbps non return to zero (NRZ) data over inter-satellite link of $3500 \mathrm{Km}$ by adopting wavelength division multiplexing (WDM) and polarization interleaving (PI) scheme. The results are reported in terms of signal to noise ratio (SNR), total received power and eye diagrams which show successful transmission of $20 \times 6 \mathrm{Gbps}$ data up to $3500 \mathrm{~km}$.
\end{abstract}

\section{Keywords}

InterSatellite Communication, High Altitude Platforms (HAP), Wavelength Division Multiplexing (WDM), Polarization Interleaving (PI),

\section{INTRODUCTION}

The evolution of optical communication system has witnessed remarkable transformation of fibres into advanced and costefficient optical wireless systems. It has also been introduced in space communications. Currently used laser satellite communication enables data transmission at the rate of several Gbps between satellites situated at a distance of hundred kilometres. This further enables the possibility of Intersatellite optical wireless communication (Is-OWC) in data transmission at much higher rates than they currently are [1]. This is achieved by integrating present OWC system with free space optics (FSO). As a result, the overall system cost reduces and access to the network increases. Larger bandwidth and higher data speed are achieved by Is-OWC system which enables to meet the increasing demand of network services [2-3]. As the recent data rates have used Inter-satellite communication up to several Tbps, Intersatellite wireless link is to be improved. Telescope gain, LT and LR are the transmitter and receiver pointing loss factor respectively. The free space path loss is explained in terms of parentheses in the above equation. Links with longer distance usually require more advanced and correct recovery, pointing and tracking system to maintain optical wireless link as the tiniest error is amplified over a link distance. So the vital requirement of Is-OWC is to align sending and receiving antenna in line of sight as small divergence can result in signal loss. One such technique used to access huge bandwidth available in optical fibre is wavelength division multiplexing (WDM). Advantages of OWC include reduction of mass and power of payload. Thus, more data rate for each watt of power can be achieved using optical beam. However, due to long separation in satellite communication optical beam has to propagate through various medium like air and vacuum. This further makes satellite communication as the most difficult area of application for optical wireless link [4]. Optical link between space communication and satellite is usually many kilometres long and though it takes place in vacuum which ought to be loss-free, there are enormous path losses. It is thus a priority issue of received optical power and very few approaches have been proposed to shrink such transmission-related losses. Optical amplification appeals to earth-to-satellite uplinks [5-9]. Researchers have also investigated the utilization of FSO for ground communication systems [10-13]. As the recent data rates have shown the use of Inter-satellite communication up to several Tbps, Intersatellite wireless link requires further improvement for better utilisation. The receiver signal power at receiver side is given by [14]:

$\mathrm{P}_{\mathrm{R}}=\mathrm{P}_{\mathrm{T}} \eta_{\mathrm{T}} \eta_{\mathrm{R}}(\lambda / 4 \pi Z)^{2} \mathrm{G}_{\mathrm{T}} \mathrm{G}_{\mathrm{R}} \mathrm{L}_{\mathrm{T}} \mathrm{L}_{\mathrm{R}}$

where $\mathrm{P}_{\mathrm{T}}$ is the transmitted optical power, $\mathrm{P}_{\mathrm{R}}$ is the received optical power, $\eta_{T}$ and $\eta_{R}$ are the optical efficiency of the transmitter and receiver respectively, $\lambda$ is the wavelength, $Z$ is the distance between the transmitter and the receiver, $\mathrm{G}_{\mathrm{T}}$ is the transmitter telescope gain, $G_{R}$ is the receiver telescope gain, $\mathrm{L}_{\mathrm{T}}$ and $\mathrm{L}_{\mathrm{R}}$ are the transmitter and receiver pointing loss factor respectively. The free space path loss is explained in terms of the parentheses in above equation. Links with longer distance usually require more advanced and correct recovery, pointing and tracking system to maintain optical wireless link as the tiniest error is amplified over a link distance. So the vital requirement of Is-OWC is to align sending and receiving antenna in line of sight as small divergence can result in signal loss. The estimated transmitting pointing loss factor is given by [14]:

$\mathrm{L}_{\mathrm{T}}=\exp \left(-\mathrm{G}_{\mathrm{T}} \theta_{\mathrm{T}}{ }^{2}\right)$

Where, $\theta_{\mathrm{T}}$ is transmitter azimuth pointing error angle, and the approximate receiver pointing error is given by the following equation [14]:

$\mathrm{L}_{\mathrm{R}}=\exp \left(-\mathrm{G}_{\mathrm{T}} \theta_{\mathrm{R}}^{2}\right)$

where, $\theta_{R}$ is the receiver azimuth pointing error. With increasing bandwidth demand to support multimedia application such as video conferencing, interest in optical fibre networks has been increased. One such technique used to 
access huge bandwidth available in optical fibre is wavelength division multiplexing (WDM) [15-17].

The remainder of paper is stated as: Section II describes the system description, Section III depicts the Result and Section followed by conclusion which is presented in Section IV.

\section{SYSTEM DESCRIPTION}

The proposed WDM-PI based Is-OWC system as illustrated in Fig. 1 is modulated in OptiSystem ${ }^{\mathrm{TM}}$ software. Six channels carrying $20 \mathrm{Gbps}$ non return to zero (NRZ) data each are modulated over light source divided into even and odd channels with aid of multiplexers. The output of multiplexers is fed to polarization controllers in order to change azimuthal parameter of even and odd channels so that adjoining channels are orthogonal to each other. Polarized signals are then multiplexed together on to a single channel and sent to receiver through an optical wireless channel (OWC). OWC consists of transmitter and receiver antenna with aperture diameter of $20 \mathrm{~cm}$ to $30 \mathrm{~cm}$ respectively. The antennas are supposed to be perfect and their optical efficiency is upheld to be 1 . Likewise, losses due to pointing error of transmitter and receiver are examined at a distance of $3500 \mathrm{~km}$. The polarization splitter (PS) is used at receiver side which divides signal according to their state of polarization (SOP) i.e. into even and odd signals. Output of PS is then de-multiplexed and perceived by photodiode trailed by a Bessel filter with a cut off frequency of $15 \mathrm{GHz}$. Fig 2 shows measured optical spectrum after modulator and single mode fiber (SMF) having span of $3500 \mathrm{Km}$.

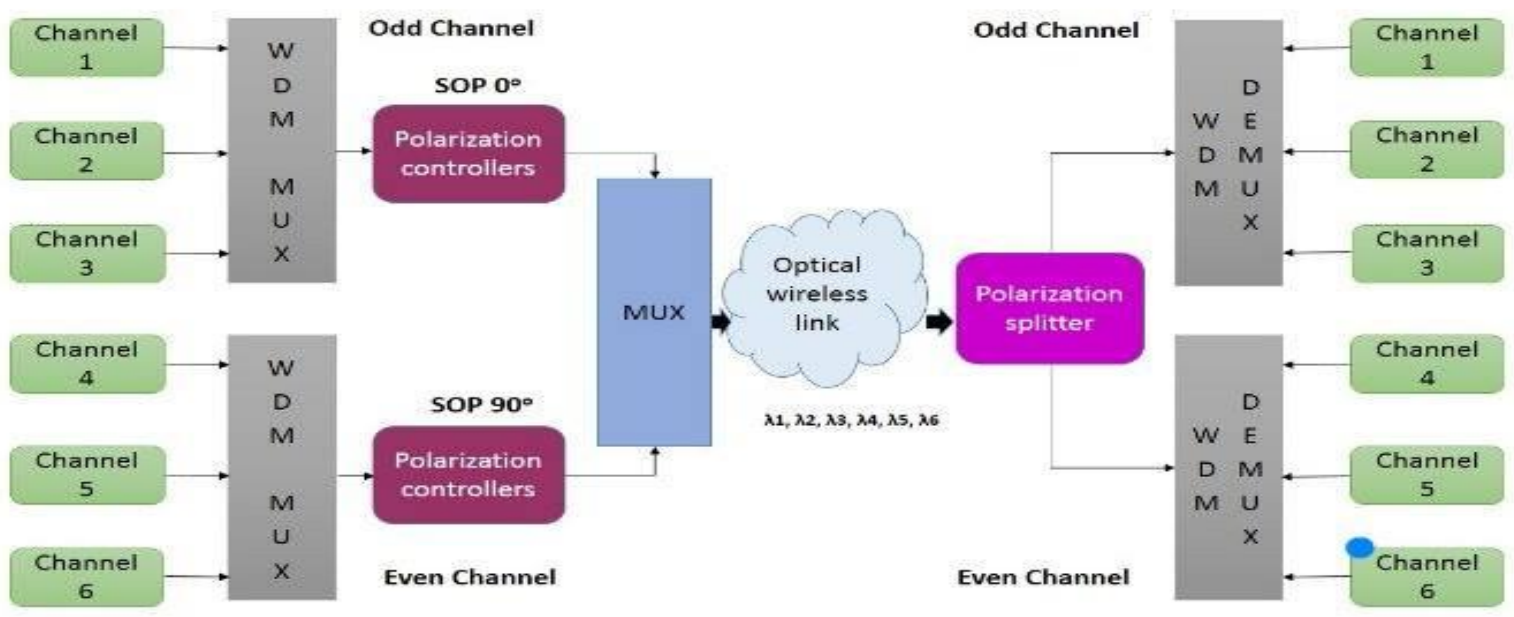

Fig.1 Proposed 6x 20Gbps Is-OWC System

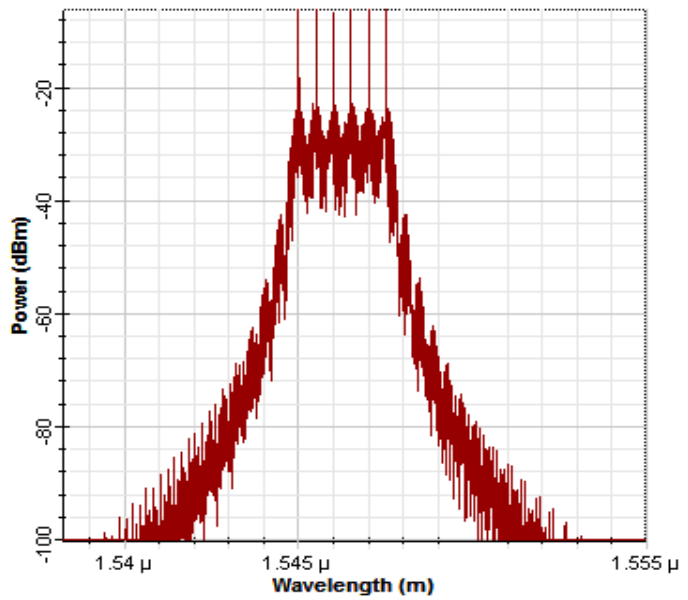

(a)

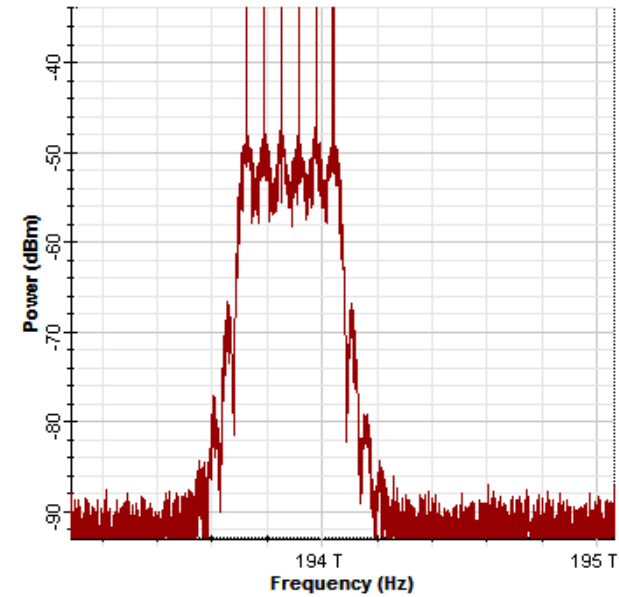

(b)

Fig. 2: Optical spectrum (a) After Multiplexer at transmission side (b) After $3500 \mathrm{Km}$ of SMF 


\section{RESULTS AND DISCUSSIONS}

In this section, results of proposed simulated setup of WDMPI based Is-OWC system are presented and discussed. Fig 3 (a) illustrates measured signal to noise ratio (SNR) and received power for channel 1, channel 2, channel 3, channel 4, channel 5 and channel 6 . The value of SNR for channel 1 is noted as $66.23 \mathrm{~dB}, 45.21 \mathrm{~dB}$ and $32.11 \mathrm{~dB}$; for channel 2 is $67.11 \mathrm{~dB}, 45.34 \mathrm{~dB}$ and $33.71 \mathrm{~dB}$; for channel 3 is $66.14 \mathrm{~dB}$, $45.11 \mathrm{~dB}$ and $33.32 \mathrm{~dB}$; for channel 4 is $66.12 \mathrm{~dB}, 46.11 \mathrm{~dB}$ and $33.43 \mathrm{~dB}$; for channel 5 is $67.21 \mathrm{~dB}, 44.21 \mathrm{~dB}$ and $33.10 \mathrm{~dB}$; for channel 6 is $66.12 \mathrm{~dB}, 41.23 \mathrm{~dB}$ and $34.22 \mathrm{~dB}$ at Is-OWC link of $500 \mathrm{~km}, 1700 \mathrm{~km}$ and $3500 \mathrm{~km}$. Similarly the received power at receiver for channel 1 is measured as $-33.50 \mathrm{dBm}$, $54.21 \mathrm{dBm}$ and $-67.12 \mathrm{dBm}$; for channel 2 is $-32.79 \mathrm{dBm}$, $54.00 \mathrm{dBm}$ and $-66.28 \mathrm{dBm}$, for channel 3 is $-33.21 \mathrm{dBm}$, $54.45 \mathrm{dBm}$ and $-66.84 \mathrm{dBm}$; for channel 4 is $-33.20 \mathrm{dBm}$, $55.11 \mathrm{dBm}$ and $-66.88 \mathrm{dBm}$; for channel 5 is $-32 \mathrm{dBm}$, $53.87 \mathrm{dBm}$ and $-66.91 \mathrm{dBm}$; for channel 6 is $-33.20 \mathrm{dBm}$,

SNR(db) Vs Range

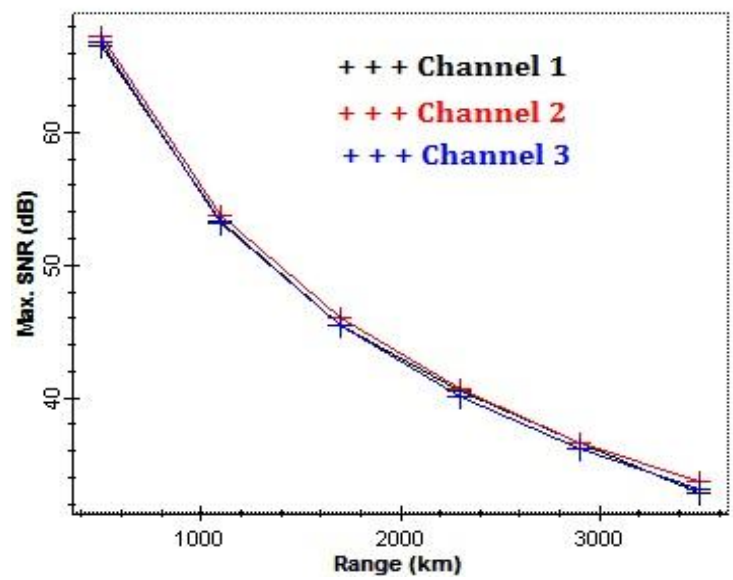

(a)

Max. Total Power $(\mathrm{dBm})$ Vs Range $(\mathrm{Km})$

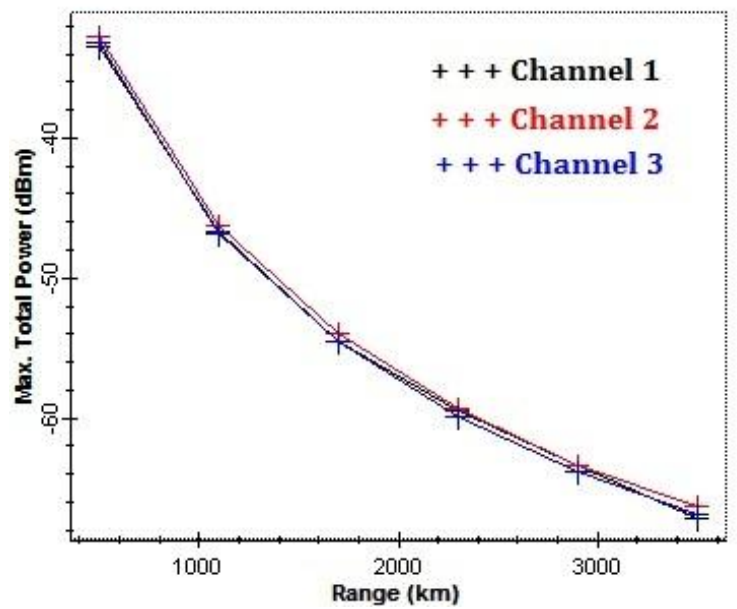

(b)
Max. SNR (dB) vs Range (Km)

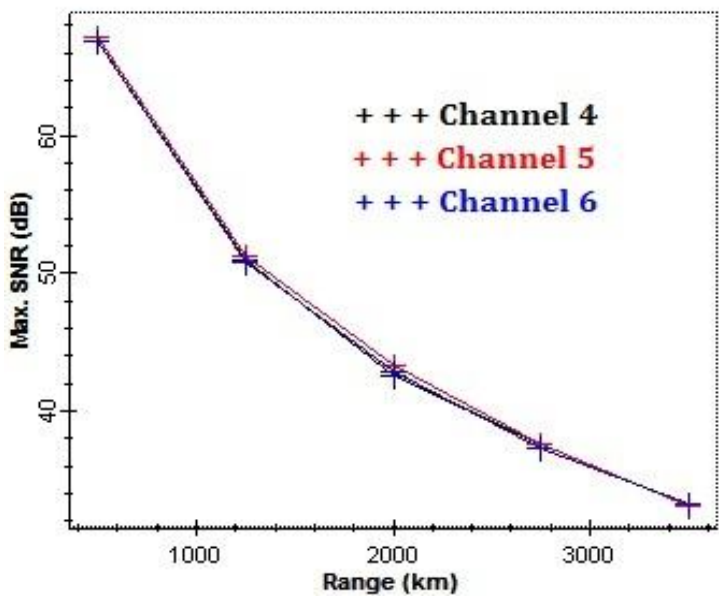

(c)

Max. Total Power $(\mathrm{dBm})$ vs Range $(\mathrm{Km})$

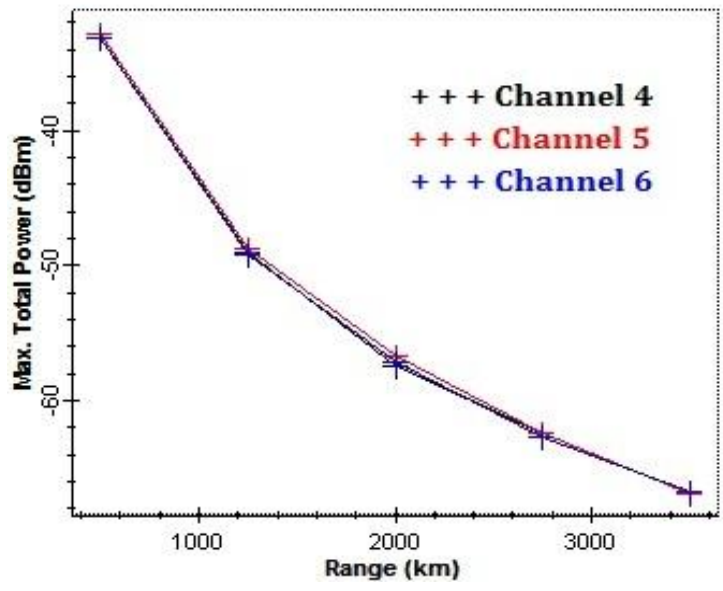

(d)

Fig 3: Measured Results (a) SNR v/s Range (b) Total Received Power (c) SNR v/s Range (d) Total Received Power

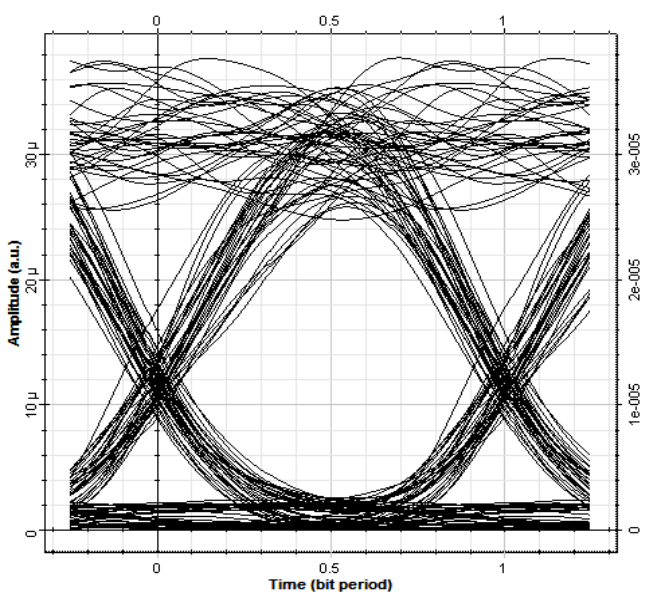

(a) 


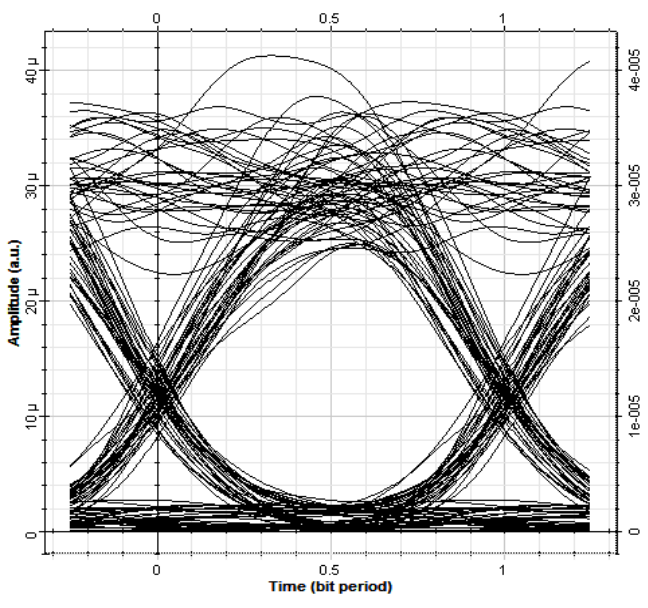

(b)

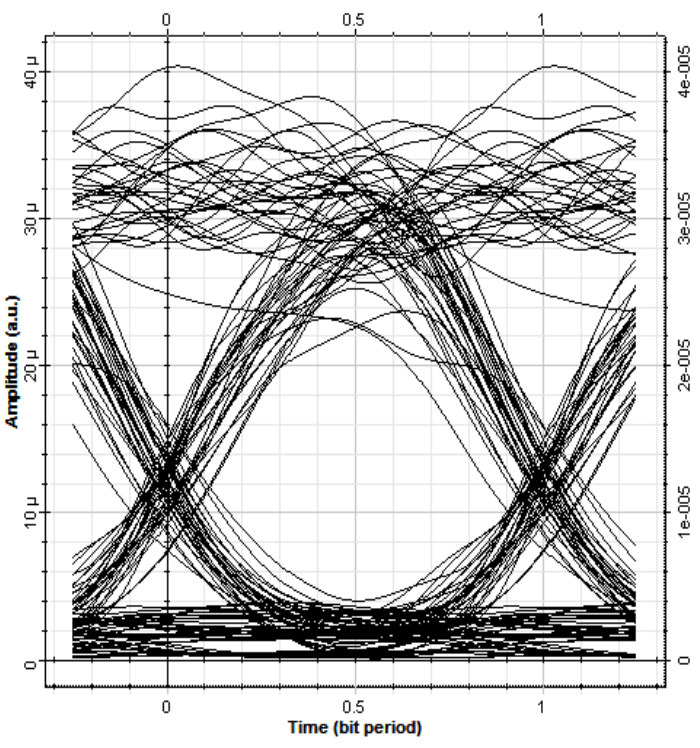

(c)

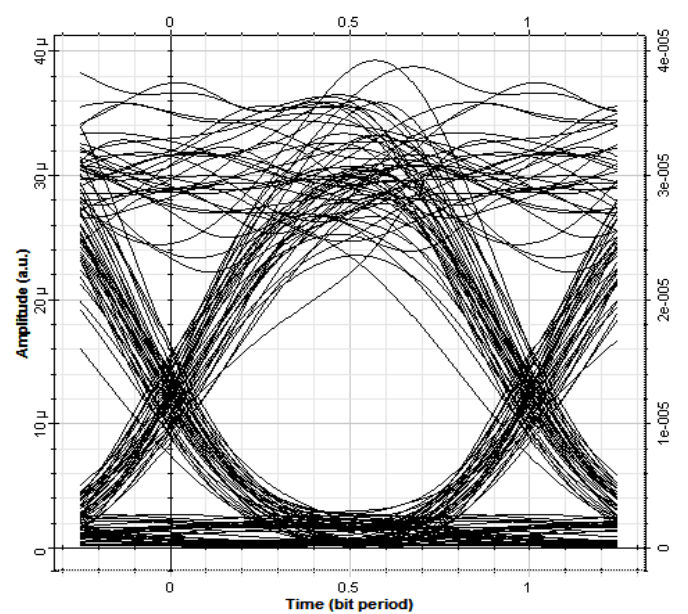

(d)

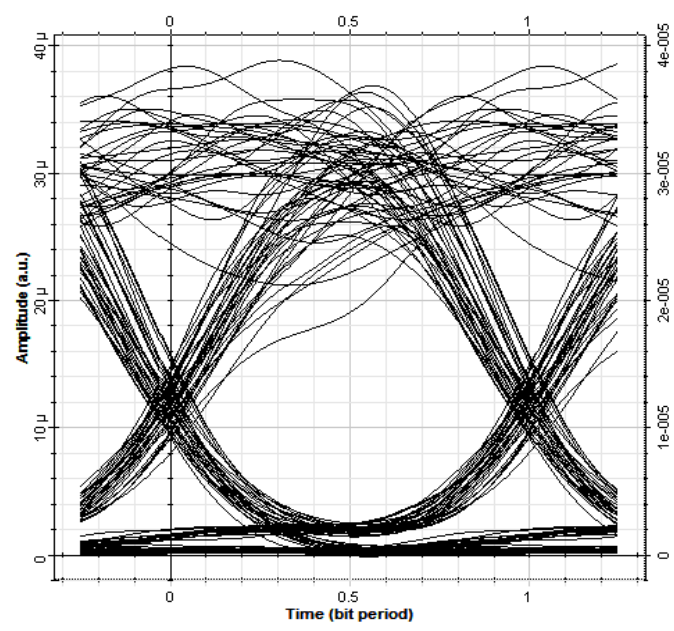

(e)

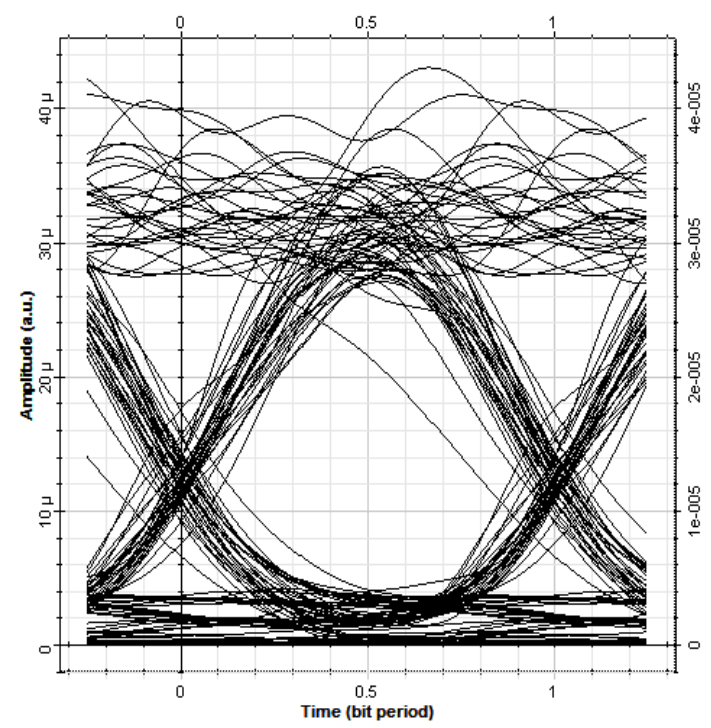

(f)

Fig 4: Eye diagrams measured at $3500 \mathrm{Km}$ (a) Channel 1 (b) Channel 2 (c) Channel 3 (d) Channel 4 (e) Channel 5 (f) Channel 6

$55.12 \mathrm{dBm}$ and $-66.76 \mathrm{dBm}$ at Is-OWC link of $500 \mathrm{~km}$, $1700 \mathrm{~km}$ and $3500 \mathrm{~km}$. Fig 4 illustrates measured eye diagram for all channels at $3500 \mathrm{~km}$ which shows that proposed IsOWC system is capable to transmit 6 X 20 Gbps data at link of $3500 \mathrm{~km}$ with acceptable SNR and total received power.

\section{CONCLUSION}

In this work, 6 channels each of which carrying $20 \mathrm{Gbps}$ are transmitted over Is-OWC link to realize total transmission of $120 \mathrm{Gbps}$ data by adopting WDM and polarization scheme. It is clear from results that all channels successfully transmitted 120 Gbps over Is-OWC link of 3500km with acceptable SNR and total received power. 


\section{REFERENCES}

[1] A. H. Hashim, "Modeling and performance study of intersatellite optical wireless communication system", International Conference on Photonics (ICP), IEEE, pp.1-4, 2010.

[2] M. A. Krainak, "Inter-satellite communications optoelectronics research at the Goddard Space Flight Center", Aerospace and Electo-system Magazine, IEEE, vol. 7, pp.44-47, 1992.

[3] N.A. Mohammed, R.A. Mohammed, H.A. Moustafa, Improved performance of M-ary PPM in different freespace optical channels due to Reed Solomon code using APD, Int. J. Sci. Eng. Res. 2 (4) (2011) 1-4.

[4] COST 297, HAPCOS - High altitude platforms for communications and other services [online], 2007, February, Available at: www.hapcos.org.

[5] A.K. Majumdar, J.C. Ricklin, Free-Space Laser Communications, Principles and Advantages, Springer Science LLC, 233 Spring Street, New York, NY 10013, USA, 2008.

[6] J. Singh, V.K. Jain, Performance analysis of BPPM and M-ary PPM optical communication systems in atmospheric turbulence, IETE Tech. Rev. 25 (4) (2008)146-153.

[7] Sushank Chaudhary and Angela Amphawan "The Role and Challenges of Free-space Optical Systems" Journal of Optical Communications. Volume 0, Issue 0, ISSN (Online) 2191-6322, ISSN (Print) 01734911, DOI: 10.1515/joc-2014-0004.

[8] E Rochat, R Dändliker, K Haroud, RH Czichy, U Roth, D Costantini, R Holzner, "Fiber amplifiers for coherent space communication", IEEE J Sel Topics Quantum Electron 7(1), 64-81 (2001).

[9] A. Polishuk, S. Arnon, "Optimization of a laser satellite communication system with an optical preamplifier", J. Optical Society of America. Vol. 21, No. 7, pp 13071315, July 2004.
[10] Sushank Chaudhary, Preety Bansal and Gurdeep Singh "Implementation of FSO Network under the Impact of Atmospheric Turbulences" International Journal of Computer Applications 75(1):34-38, August 2013.

[11] Vishal Sharma and Sushank Chaudhary "Implementation of Hybrid OFDM-FSO Transmission System" International Journal of Computer Applications 58(8):3740, November 2012.

[12] Sushank Chaudhary, Angela Amphawan, Kashif Nisar, "Realization of free space optics with OFDM under atmospheric turbulence", Optik - International Journal for Light and Electron Optics, Available online 8 July 2014, ISSN 0030-4026

[13] Vishal Sharma, Sushank, "High speed CO-OFDM-FSO transmission system", Optik - International Journal for Light and Electron Optics, Volume 125, Issue 6, March 2014, Pages 1761-1763, ISSN 0030-4026

[14] A. Polishuk, S. Arnon, 'Optimization of a laser satellite communication system with an optical preamplifier', J. Optical Society of America. Vol. 21, No. 7, pp $1307-$ 1315, July 2004.

[15] Sushank Chaudhary, Preety Bansal and Manisha Lumb "Effect of Beam Divergence on WDM-FSO Transmission System" International Journal of Computer Applications 93(1):28-32, May 2014.

[16] Ramandeep Kaur and Sushank Chaudhary "Simulative Investigation of Laser Line-width and Channel Spacing for Realization of DWDM Systems under the Impact of Four Wave Mixing" Journal of Optical Communications. Volume 35, Issue 2, Pages 157-165, ISSN (Online) 2191-6322, ISSN (Print) 01734911, DOI: 10.1515/joc2013-0152, March 2014

[17] Sushank Chaudhary, Saurabh Sharma and Praveen "Role of Turbulences in WDM-Polarisation Interleaving Scheme based Inter-Satellite Communication system" International Journal of Computer Applications Vol. 104 - No 10, Oct. 2014. 